\title{
Circuit
}

Musiques contemporaines

\section{Introduction : l'arbre et le fleuve}

\section{Jonathan Goldman}

Volume 20, numéro 3, 2010

Gilles Tremblay, ou le plain-chant contemporain

URI : https://id.erudit.org/iderudit/044858ar

DOI : https://doi.org/10.7202/044858ar

Aller au sommaire du numéro

Éditeur(s)

Les Presses de l'Université de Montréal

ISSN

1183-1693 (imprimé)

1488-9692 (numérique)

Découvrir la revue

Citer ce document

Goldman, J. (2010). Introduction : l'arbre et le fleuve. Circuit, 20(3), 5-7. https://doi.org/10.7202/044858ar

Ce document est protégé par la loi sur le droit d'auteur. L'utilisation des services d'Érudit (y compris la reproduction) est assujettie à sa politique d'utilisation que vous pouvez consulter en ligne.

https://apropos.erudit.org/fr/usagers/politique-dutilisation/
Cet article est diffusé et préservé par Érudit.

Érudit est un consortium interuniversitaire sans but lucratif composé de l’Université de Montréal, l'Université Laval et l'Université du Québec à Montréal. Il a pour mission la promotion et la valorisation de la recherche. https://www.erudit.org/fr/ 


\section{Introduction : \\ l'arbre et le fleuve}

Jonathan Goldman

Dans un court texte récent à propos de Gilles Tremblay, Walter Boudreau a évoqué pour le décrire l'image d'un arbre' «aux racines profondes, solidement ancré dans un riche terreau... ». Tremblay, dont la carrière est contemporaine au développement de la musique de création au Québec, a contribué à notre paysage musical en cultivant des œuvres fortes et durables. Il relevait donc de l'évidence de consacrer une édition de la Série Hommage, cet immense «méta-événement» chapeauté par la Société québécoise de musique contemporaine (SMCQ), à Tremblay. L'édition 2009-2010 de cette série a réuni plusieurs dizaines de concerts, des spectacles pour la jeunesse et des événements divers qui ont eu lieu à travers le Québec, dans les grandes salles de concert, dans les écoles, et dans le cas d'un événement réalisé par Circuit, dans les passages souterrains de Montréal ${ }^{2}$. Or, avouons-le sans gêne, cette Série Hommage constitue un véritable assaut médiatique. Elle atteint un auditoire qui décuple largement le public habituel de la musique contemporaine: selon la SMCQ, 40 ooo personnes ont entendu au moins une œuvre de Tremblay au cours de la saison?3.

Si Gilles Tremblay est pareil à un arbre, il est aussi semblable à un fleuve, ce grand symbole du pays qui est le nôtre, à travers la métonymie qui relie le Saint-Laurent au Québec tout entier. De façon analogue, on pourrait évoquer Tremblay en rappelant Fleuves (1976), son œuvre magistrale pour orchestre. L'arbre et le fleuve: deux images contrastantes pour tenter de circonscrire les visages multiples de ce musicien protéiforme.

Un de ses visages est celui du grand pédagogue qui a formé plusieurs générations de musiciens au Conservatoire de musique de Montréal, où il a inlassablement encouragé ses élèves à trouver leurs propres chemins - tributaires du fleuve pour ainsi dire - ainsi qu'à aller à contre-courant, tels des
1. www.smcq.qc.ca/smcq/fr/ hommage/2009/mot/

2. Dans le cadre de l'événement «Art Souterrain ", lors de la Nuit blanche de Montréal, le 27 février 2010, Circuit fêtait ses 20 ans en diffusant deux œuvres électroniques qui ont été commandées pour l'Expo 67 : Centre-élan (1967) de Gilles Tremblay (sonorisation du Pavillon du Québec) et Katimavik (1967) d'Otto Joachim (sonorisation du Pavillon du Canada). 3. www.smcq.qc.ca/smcq/fr/ hommage/2009/ 
4. Jean Boivin, La Classe de Messiaen, Paris, Bourgois, 1995

5. Voir à ce propos la critique de Christophe Huss, publiée dans Le Devoir du 20 novembre 2009 (www.ledevoir. ca/culture/musique/277614/musiqueclassique-feerie-visuelle) ainsi que la lettre ouverte que Michel Gonneville lui a adressée (www.chantslibres.org/fr/ nouvelles/10013.php).

6. Lors de la journée d'étude sur Gilles Tremblay organisée par Sylvain Caron et Marie-Thérèse Lefebvre sous l'égide de la Société québécoise de recherche sur la musique. saumons. Dans ce numéro, Danick Trottier mène une enquête auprès de plusieurs générations des anciens élèves de Tremblay, en suivant le modèle de l'ouvrage de Jean Boivin ${ }^{4}$ à propos d'une autre classe mythique d'analyse musicale, celle d'Olivier Messiaen, où, justement, se trouvait le jeune Gilles Tremblay. Trottier entreprend ce projet dans l'optique d'élucider le contenu même de l'enseignement de Tremblay, qui a tant inspiré les compositeurs québécois jusqu'à ce jour. C'est d'ailleurs les propos recueillis dans cette enquête qui sont à l'origine de la référence au plain-chant dans le titre choisi pour ce numéro. En effet, il semblerait que Tremblay faisait de nombreuses allusions au chant grégorien dans ses cours, tel que relaté par certains de ses anciens élèves.

Bien sûr, précisons immédiatement que Gilles Tremblay n’était pas seulement l'objet de la Série Hommage de cette année, mais il a également agi comme vecteur de création au sein de celle-ci, comme en témoigne l'éblouissante éclosion (si vous permettez qu'une métaphore agricole s'ajoute aux autres, aquatique et arborescente, déjà évoquées...) d'un opéra, le premier dans son catalogue. En effet, l'événement qui a couronné la saison a certainement été L'Eau qui danse, la Pomme qui chante et l'Oiseau qui dit la vérité, cette coproduction de Chants libres et du Nouvel Ensemble Moderne (NEM) qui a eu lieu au Monument-National en novembre 2009, et qui sera traité en profondeur dans l'article de la musicologue française Cécile Auzolle, qui l'inscrit dans la thématique du merveilleux. Même si la critique musicale montréalaise a quelque peu boudé l'opéra, telle qu'est son habitude lorsqu'il est question de musique contemporaine, nombreux sont ceux qui s'accordent à dire que l'œuvre a été une réussite musicale, théâtrale, esthétique, et ce, auprès d'un large publics.

Mais, au-delà de la Série Hommage, qui est l'occasion de ce numéro, nous souhaitons ici toucher aussi à une exégèse de la spécificité du langage musical propre à Gilles Tremblay. Trop rares sont les vues synthétiques de l'œuvre de compositeurs d'aujourd'hui, et c'est pourquoi nous sommes si contents d'accueillir le projet ambitieux de Serge Provost dans le Cahier d'analyse de ce numéro. Provost tâche de distiller, à partir d'exemples tirés de quelques œuvres-clés comme Fleuves ou Réseaux, les principes d'une poétique musicale chez Tremblay. Si Provost remonte des réalisations musicales aux idées qui en sont à l'origine, Vincent Ranallo, baryton et interprète de choix des œuvres vocales de Tremblay, se penche pour sa part sur Oralléluiants (1975), une des œuvres les plus caractéristiques de Tremblay, en soulignant la dimension spirituelle de ses timbres étincelants ${ }^{6}$. 
La rubrique Document laisse la parole à Gilles Tremblay, en présentant un entretien réalisé par Jean Lesage en 1997 avec Tremblay dans lequel celui-ci évoque son enfance, sa formation auprès de Claude Champagne, ses rencontres avec Edgard Varèse, sa vie de jeune compositeur dans le Montréal des années 1950 et 1960, sa rencontre avec Messiaen et Yvonne Loriod, et dans lequel il livre ses réflexions sur sa philosophie de l'enseignement de la musique.

Pour sa part, Marie-Thérèse Lefebvre tisse des liens entre ce numéro et deux autres qui font partie du passé de Circuit: le vol. 5, no 1 (1994) et le vol. 6, nº 1 (1995), tous deux dirigés par ses soins. Dans le présent numéro, elle fournit dans la rubrique Annexe des compléments bio-bibliographiques qui seront d'une précieuse utilité pour les chercheurs futurs. Ces compléments s'ajoutent aux dossiers analogues se trouvant dans les deux numéros mentionnés précédemment qui sont, soit dit en passant, désormais disponibles gratuitement sur le réseau Érudit, comme tous les numéros de Circuit qui datent de plus de deux ans7. Les illustrations qui ornent le présent numéro sont tirées d'une exposition en hommage à Tremblay qui a été montée par le collectif ARPRIM dans le cadre de la Série Hommage. Enfin, nous sommes ravis d'accueillir Maxime McKinley, notre nouveau directeur administratif et secrétaire de rédaction, au sein de notre équipe.

Bonne lecture!
7. www.erudit.org/revue/circuit/1994/v5/ n1/index.html et www.erudit.org/revue/ circuit/1995/v6/n1/index.html/

Montréal, juillet 2010 